\title{
Numerical radius inequalities for Hilbert space operators. II
}

\author{
by \\ Mohammad El-Haddad (Dammam) and Fuad Kittaneh (Amman)
}

\begin{abstract}
We give several sharp inequalities involving powers of the numerical radii and the usual operator norms of Hilbert space operators. These inequalities, which are based on some classical convexity inequalities for nonnegative real numbers and some operator inequalities, generalize earlier numerical radius inequalities.
\end{abstract}

1. Introduction. Let $B(H)$ denote the $C^{*}$-algebra of all bounded linear operators on a complex Hilbert space $H$ with inner product $\langle\cdot, \cdot\rangle$. For $A \in$ $B(H)$, let $w(A)$ and $\|A\|$ denote the numerical radius and the usual operator norm of $A$, respectively. It is well known that $w(\cdot)$ defines a norm on $B(H)$, which is equivalent to the usual operator norm $\|\cdot\|$. In fact, for every $A \in$ $B(H)$,

$$
\frac{1}{2}\|A\| \leq w(A) \leq\|A\| .
$$

The inequalities in (1) are sharp. The first inequality becomes an equality if $A^{2}=0$. The second inequality becomes an equality if $A$ is normal. For basic properties of the numerical radius, we refer to [3] and [4]. The inequalities in (1) have been improved considerably by the second author in [8] and [9]. It has been shown in [8] and [9], respectively, that if $A \in B(H)$, then

$$
w(A) \leq \frac{1}{2}\left\||A|+\left|A^{*}\right|\right\| \leq \frac{1}{2}\left(\|A\|+\left\|A^{2}\right\|^{1 / 2}\right),
$$

where $|A|=\left(A^{*} A\right)^{1 / 2}$ is the absolute value of $A$, and

$$
\frac{1}{4}\left\|A^{*} A+A A^{*}\right\| \leq w^{2}(A) \leq \frac{1}{2}\left\|A^{*} A+A A^{*}\right\| .
$$

The inequalities in (2), which refine the second inequality in (1), have been utilized in [8] to derive an estimate for the numerical radius of the Frobenius companion matrix. Such an estimate can be employed to give new bounds for the zeros of polynomials (see, e.g., [7], [8], and references therein).

If $A=B+i C$ is the Cartesian decomposition of $A$, then $B$ and $C$ are self-adjoint, and $A^{*} A+A A^{*}=2\left(B^{2}+C^{2}\right)$. Thus, the inequalities in (3) can

2000 Mathematics Subject Classification: 47A12, 47A30, 47A63, 47B15.

Key words and phrases: numerical radius, operator norm, Cartesian decomposition, Jensen's inequality, mixed Schwarz inequality, triangle inequality. 
be written as

$$
\frac{1}{2}\left\|B^{2}+C^{2}\right\| \leq w^{2}(A) \leq\left\|B^{2}+C^{2}\right\|,
$$

or equivalently, as

$$
\frac{1}{4}\left\|(B+C)^{2}+(B-C)^{2}\right\| \leq w^{2}(A) \leq \frac{1}{2}\left\|(B+C)^{2}+(B-C)^{2}\right\| .
$$

The purpose of this paper is to establish considerable generalizations of these inequalities that are based on some classical convexity inequalities for nonnegative real numbers and some operator inequalities. Usual operator norm inequalities and a related numerical radius inequality for the sum of two operators are also presented.

Other recent numerical radius inequalities have been obtained by Dragomir [2] and Yamazaki [10]. The inequalities in [2] are related to the Euclidean radius of two Hilbert space operators, and those in [10] involve the Aluthge transform.

2. Generalized numerical radius inequalities. To prove our generalized numerical radius inequalities, we need several well known lemmas. The first lemma is a simple consequence of the classical Jensen inequality concerning the convexity or concavity of certain power functions. It is a special case of Schlömilch's inequality for weighted means of nonnegative real numbers (see, e.g., [5, p. 26]).

Lemma 1. For $a, b \geq 0,0<\alpha<1$, and $r \neq 0$, let $M_{r}(a, b, \alpha)=$ $\left(\alpha a^{r}+(1-\alpha) b^{r}\right)^{1 / r}$ and let $M_{0}(a, b, \alpha)=a^{\alpha} b^{1-\alpha}$. Then

$$
M_{r}(a, b, \alpha) \leq M_{s}(a, b, \alpha) \quad \text { for } r \leq s .
$$

The second lemma is another application of Jensen's inequality (see, e.g., [5, p. 28]).

Lemma 2. For $a, b \geq 0$, and $r>0$, let $N_{r}(a, b)=\left(a^{r}+b^{r}\right)^{1 / r}$. Then

$$
N_{s}(a, b) \leq N_{r}(a, b) \quad \text { for } s \geq r>0 .
$$

The third lemma follows from the spectral theorem for positive operators and Jensen's inequality (see e.g., [6]).

Lemma 3. Let $A \in B(H)$ be positive, and let $x \in H$ be any unit vector. Then

(a) $\langle A x, x\rangle^{r} \leq\left\langle A^{r} x, x\right\rangle$ for $r \geq 1$.

(b) $\left\langle A^{r} x, x\right\rangle \leq\langle A x, x\rangle^{r}$ for $0<r \leq 1$.

The fourth lemma is an immediate consequence of the spectral theorem for self-adjoint operators. For generalizations of this lemma, we refer to [6]. 
Lemma 4. Let $A \in B(H)$ be self-adjoint, and let $x \in H$ be any vector. Then

$$
|\langle A x, x\rangle| \leq\langle|A| x, x\rangle .
$$

The fifth lemma is known as the generalized mixed Schwarz inequality (see, e.g., [6]).

Lemma 5. Let $A \in B(H)$, and $0 \leq \alpha \leq 1$. Then

$$
|\langle A x, y\rangle|^{2} \leq\left\langle|A|^{2 \alpha} x, x\right\rangle\left\langle\left|A^{*}\right|^{2(1-\alpha)} y, y\right\rangle \quad \text { for all } x, y \in H .
$$

The sixth lemma is a subadditivity norm inequality for fractional powers of positive operators, which has been proved in [1].

Lemma 6. Let $A, B \in B(H)$ be positive. Then

$$
\left\|(A+B)^{r}\right\| \leq\left\|A^{r}+B^{r}\right\| \quad \text { for } 0<r \leq 1 .
$$

Our first result is a generalization of the first inequality in (2).

Theorem 1. Let $A \in B(H), 0<\alpha<1$, and $r \geq 1$. Then

$$
w^{r}(A) \leq \frac{1}{2}\left\||A|^{2 \alpha r}+\left|A^{*}\right|^{2(1-\alpha) r}\right\| .
$$

Proof. For every unit vector $x \in H$, we have

$$
\begin{array}{rlrl}
|\langle A x, x\rangle| & \leq\left\langle|A|^{2 \alpha} x, x\right\rangle^{1 / 2}\left\langle\left|A^{*}\right|^{2(1-\alpha)} x, x\right\rangle^{1 / 2} & \quad \text { (by Lemma 5) } \\
& \leq\left(\frac{\left\langle|A|^{2 \alpha} x, x\right\rangle^{r}+\left\langle\left|A^{*}\right|^{2(1-\alpha)} x, x\right\rangle^{r}}{2}\right)^{1 / r} & \text { (by Lemma 1) } \\
& \leq\left(\frac{\left\langle|A|^{2 \alpha r} x, x\right\rangle+\left\langle\left|A^{*}\right|^{2(1-\alpha) r} x, x\right\rangle}{2}\right)^{1 / r} \quad \text { (by Lemma 3(a)). }
\end{array}
$$

Thus,

$$
|\langle A x, x\rangle|^{r} \leq \frac{1}{2}\left\langle\left(|A|^{2 \alpha r}+\left|A^{*}\right|^{2(1-\alpha) r}\right) x, x\right\rangle
$$

and so

$$
\begin{aligned}
w^{r}(A) & =\sup \left\{|\langle A x, x\rangle|^{r}: x \in H,\|x\|=1\right\} \\
& \leq \frac{1}{2} \sup \left\{\left\langle\left(|A|^{2 \alpha r}+\left|A^{*}\right|^{2(1-\alpha) r}\right) x, x\right\rangle: x \in H,\|x\|=1\right\} \\
& =\frac{1}{2}\left\||A|^{2 \alpha r}+\left|A^{*}\right|^{2(1-\alpha) r}\right\|,
\end{aligned}
$$

as required.

Our second result is a generalization of the second inequality in (3).

Theorem 2. Let $A \in B(H), 0<\alpha<1$, and $r \geq 1$. Then

$$
w^{2 r}(A) \leq\left\|\alpha|A|^{2 r}+(1-\alpha)\left|A^{*}\right|^{2 r}\right\| .
$$


Proof. For every unit vector $x \in H$, we have

$$
\begin{aligned}
|\langle A x, x\rangle|^{2} & \leq\left\langle|A|^{2 \alpha} x, x\right\rangle\left\langle\left|A^{*}\right|^{2(1-\alpha)} x, x\right\rangle \quad \text { (by Lemma 5) } \\
& \leq\left\langle|A|^{2} x, x\right\rangle^{\alpha}\left\langle\left|A^{*}\right|^{2} x, x\right\rangle^{1-\alpha} \quad(\text { by Lemma } 3(\mathrm{~b})) \\
& \leq\left(\alpha\left\langle|A|^{2} x, x\right\rangle^{r}+(1-\alpha)\left\langle\left|A^{*}\right|^{2} x, x\right\rangle^{r}\right)^{1 / r} \quad \text { (by Lemma 1) } \\
& \leq\left(\alpha\left\langle|A|^{2 r} x, x\right\rangle+(1-\alpha)\left\langle\left|A^{*}\right|^{2 r} x, x\right\rangle\right)^{1 / r} \quad \text { (by Lemma 3(a)) } \\
& =\left\langle\left(\alpha|A|^{2 r}+(1-\alpha)\left|A^{*}\right|^{2 r}\right) x, x\right\rangle^{1 / r} .
\end{aligned}
$$

Thus,

$$
|\langle A x, x\rangle|^{2 r} \leq\left\langle\left(\alpha|A|^{2 r}+(1-\alpha)\left|A^{*}\right|^{2 r}\right) x, x\right\rangle,
$$

and so

$$
\begin{aligned}
w^{2 r}(A) & =\sup \left\{|\langle A x, x\rangle|^{2 r}: x \in H,\|x\|=1\right\} \\
& \leq \sup \left\{\left\langle\left(\alpha|A|^{2 r}+(1-\alpha)\left|A^{*}\right|^{2 r}\right) x, x\right\rangle: x \in H,\|x\|=1\right\} \\
& =\left\|\alpha|A|^{2 r}+(1-\alpha)\left|A^{*}\right|^{2 r}\right\|,
\end{aligned}
$$

as required.

Our next two results are generalizations of the second inequality in (4).

Theorem 3. Let $A \in B(H)$ with the Cartesian decomposition $A=$ $B+i C$, and let $0<r \leq 2$. Then

$$
w^{r}(A) \leq\left\||B|^{r}+|C|^{r}\right\| .
$$

Proof. First we prove an inequality stronger than (7) for the special case where $1 \leq r \leq 2$. For every unit vector $x \in H$, and for $1 \leq r \leq 2$, we have

$$
\begin{aligned}
& |\langle A x, x\rangle|=\left(\langle B x, x\rangle^{2}+\langle C x, x\rangle^{2}\right)^{1 / 2} \\
& \leq\left(|\langle B x, x\rangle|^{r}+|\langle C x, x\rangle|^{r}\right)^{1 / r} \quad \text { (by Lemma 2) } \\
& \leq\left(\langle|B| x, x\rangle^{r}+\langle|C| x, x\rangle^{r}\right)^{1 / r} \quad \text { (by Lemma 4) } \\
& \leq\left(\left\langle|B|^{r} x, x\right\rangle+\left\langle|C|^{r} x, x\right\rangle\right)^{1 / r} \quad \text { (by Lemma 3(a)) } \\
& =\left\langle\left(|B|^{r}+|C|^{r}\right) x, x\right\rangle^{1 / r} \text {. }
\end{aligned}
$$

Thus, we obtain the stronger inequality

$$
|\langle A x, x\rangle|^{r} \leq\left\langle\left(|B|^{r}+|C|^{r}\right) x, x\right\rangle,
$$

and so

$$
\begin{aligned}
w^{r}(A) & =\sup \left\{|\langle A x, x\rangle|^{r}: x \in H,\|x\|=1\right\} \\
& \leq \sup \left\{\left\langle\left(|B|^{r}+|C|^{r}\right) x, x\right\rangle: x \in H,\|x\|=1\right\} \\
& =\left\||B|^{r}+|C|^{r}\right\| .
\end{aligned}
$$


For the general case, where $0<r \leq 2$, we have

$$
\begin{aligned}
w^{r}(A) & \leq\left\|B^{2}+C^{2}\right\|^{r / 2} \quad \text { (by the second inequality in (4)) } \\
& =\left\|\left(B^{2}+C^{2}\right)^{r / 2}\right\| \\
& \left.\leq\left\||B|^{r}+|C|^{r}\right\| \quad \text { (by Lemma } 6\right)
\end{aligned}
$$

as required.

Theorem 4. Let $A \in B(H)$ with the Cartesian decomposition $A=$ $B+i C$, and let $r \geq 2$. Then

$$
w^{r}(A) \leq 2^{r / 2-1}\left\||B|^{r}+|C|^{r}\right\| .
$$

Proof. For every unit vector $x \in H$, we have

$$
\begin{aligned}
& \frac{|\langle A x, x\rangle|}{\sqrt{2}}=\left(\frac{\langle B x, x\rangle^{2}+\langle C x, x\rangle^{2}}{2}\right)^{1 / 2} \\
& \leq\left(\frac{|\langle B x, x\rangle|^{r}+|\langle C x, x\rangle|^{r}}{2}\right)^{1 / r} \quad \text { (by Lemma 1) } \\
& \leq 2^{-1 / r}\left(\langle|B| x, x\rangle^{r}+\langle|C| x, x\rangle^{r}\right)^{1 / r} \quad \text { (by Lemma 4) } \\
& \leq 2^{-1 / r}\left(\left\langle|B|^{r} x, x\right\rangle+\left\langle|C|^{r} x, x\right\rangle\right)^{1 / r} \quad \text { (by Lemma } 3(\mathrm{a}) \text { ) } \\
& =2^{-1 / r}\left\langle\left(|B|^{r}+|C|^{r}\right) x, x\right\rangle^{1 / r} \text {. }
\end{aligned}
$$

Thus,

$$
|\langle A x, x\rangle|^{r} \leq 2^{r / 2-1}\left\langle\left(|B|^{r}+|C|^{r}\right) x, x\right\rangle,
$$

and so

$$
\begin{aligned}
w^{r}(A) & =\sup \left\{|\langle A x, x\rangle|^{r}: x \in H,\|x\|=1\right\} \\
& \leq 2^{r / 2-1} \sup \left\{\left\langle\left(|B|^{r}+|C|^{r}\right) x, x\right\rangle: x \in H,\|x\|=1\right\} \\
& =2^{r / 2-1}\left\||B|^{r}+|C|^{r}\right\|,
\end{aligned}
$$

as required.

Our final result in this section is a generalization of the inequalities in (5).

Theorem 5. Let $A \in B(H)$ with the Cartesian decomposition $A=$ $B+i C$, and let $r \geq 2$. Then

$$
2^{-r / 2-1}\left\||B+C|^{r}+|B-C|^{r}\right\| \leq w^{r}(A) \leq \frac{1}{2}\left\||B+C|^{r}+|B-C|^{r}\right\| .
$$

Proof. As in the proof of the first inequality in (3) (see Theorem 1 in [9]), we have

$$
w^{2}(A) \geq \frac{1}{2}\left\|(B \pm C)^{2}\right\|
$$

Thus,

$$
w^{r}(A) \geq 2^{-r / 2}\left\|(B \pm C)^{2}\right\|^{r / 2}=2^{-r / 2}\left\||B \pm C|^{r}\right\|,
$$


and so

$$
\begin{aligned}
2 w^{r}(A) & \geq 2^{-r / 2}\left(\left\||B+C|^{r}\right\|+\left\||B-C|^{r}\right\|\right) \\
& \geq 2^{-r / 2}\left\||B+C|^{r}+|B-C|^{r}\right\| \quad \text { (by the triangle inequality). }
\end{aligned}
$$

Hence,

$$
w^{r}(A) \geq 2^{-r / 2-1}\left\||B+C|^{r}+|B-C|^{r}\right\|,
$$

which proves the first inequality in (8). To prove the second inequality in (8), let $x \in H$ be any unit vector. Then

$$
\begin{aligned}
|\langle A x, x\rangle|^{r}= & \left(\langle B x, x\rangle^{2}+\langle C x, x\rangle^{2}\right)^{r / 2} \\
= & 2^{-r / 2}\left(\langle(B+C) x, x\rangle^{2}+\langle(B-C) x, x\rangle^{2}\right)^{r / 2} \\
\leq & 2^{-r / 2} 2^{r / 2-1}\left(|\langle(B+C) x, x\rangle|^{r}+|\langle(B-C) x, x\rangle|^{r}\right) \\
& \quad \quad\left(\text { by the convexity of the function } f(t)=t^{r / 2} \text { on }[0, \infty)\right) \\
\leq & \frac{1}{2}\left(\langle|B+C| x, x\rangle^{r}+\langle|B-C| x, x\rangle^{r}\right) \quad(\text { by Lemma } 4) \\
\leq & \frac{1}{2}\left(\left\langle|B+C|^{r} x, x\right\rangle+\left\langle|B-C|^{r} x, x\right\rangle\right) \quad(\text { by Lemma } 3(\text { a) }) \\
= & \frac{1}{2}\left\langle\left(|B+C|^{r}+|B-C|^{r}\right) x, x\right\rangle .
\end{aligned}
$$

Now,

$$
\begin{aligned}
w^{r}(A) & =\sup \left\{|\langle A x, x\rangle|^{r}: x \in H,\|x\|=1\right\} \\
& \leq \frac{1}{2} \sup \left\{\left\langle\left(|B+C|^{r}+|B-C|^{r}\right) x, x\right\rangle^{r}: x \in H,\|x\|=1\right\} \\
& =\frac{1}{2}\left\||B+C|^{r}+|B-C|^{r}\right\|,
\end{aligned}
$$

which proves the second inequality in (8), and completes the proof of the theorem.

In view of the first inequality in (5) and the second inequality in (8), one might conjecture that the factor $2^{-r / 2-1}$ in the first inequality in (8) can be replaced by the absolute constant $1 / 4$. However, this is refuted by the two-dimensional example $A=\left[\begin{array}{ll}0 & 2 \\ 0 & 0\end{array}\right]$. In this case,

$$
\frac{1}{4}\left\||B+C|^{r}+|B-C|^{r}\right\|=2^{r / 2-1}>1=w^{r}(A) \quad \text { for } r>2 .
$$

This example also shows that the first inequality in (8) is sharp. In fact, this inequality becomes an equality for every operator $A$ with $A^{2}=0$. To see this, we invoke Corollary 1 in [8] and Proposition 1 in [9], where it has been shown that if $A^{2}=0$, then $w(A)=\frac{1}{2}\|A\|$ and $\left\|A^{*} A+A A^{*}\right\|=\|A\|^{2}$. Also, if $A^{2}=0$, then by simple computations,

$$
\left\||B+C|^{r}+|B-C|^{r}\right\|=2^{-r / 2+1}\left\|A^{*} A+A A^{*}\right\|^{r / 2}=2^{-r / 2+1}\|A\|^{r} .
$$

Thus,

$$
2^{-r / 2-1}\left\||B+C|^{r}+|B-C|^{r}\right\|=2^{-r}\|A\|^{r}=w^{r}(A) .
$$


3. Inequalities for sums of operators. In this section, we present usual operator norm inequalities and a related numerical radius inequality for the sum of two operators.

Theorem 6. Let $A, B \in B(H), 0<\alpha<1$, and $r \geq 1$. Then

$$
\|A+B\|^{r} \leq 2^{r-2}\left(\left\||A|^{2 \alpha r}+|B|^{2 \alpha r}\right\|+\left\|\left|A^{*}\right|^{2(1-\alpha) r}+\left|B^{*}\right|^{2(1-\alpha) r}\right\|\right) .
$$

Proof. For any unit vectors $x, y \in H$, we have

$$
\begin{aligned}
& |\langle(A+B) x, y\rangle| \leq|\langle A x, y\rangle|+|\langle B x, y\rangle| \quad \text { (by the triangle inequality) } \\
& \leq\left\langle|A|^{2 \alpha} x, x\right\rangle^{1 / 2}\left\langle\left|A^{*}\right|^{2(1-\alpha)} y, y\right\rangle^{1 / 2}+\left\langle|B|^{2 \alpha} x, x\right\rangle^{1 / 2}\left\langle\left|B^{*}\right|^{2(1-\alpha)} y, y\right\rangle^{1 / 2}
\end{aligned}
$$

(by Lemma 5)

$$
\leq\left(\frac{\left\langle|A|^{2 \alpha} x, x\right\rangle^{r}+\left\langle\left|A^{*}\right|^{2(1-\alpha)} y, y\right\rangle^{r}}{2}\right)^{1 / r}+\left(\frac{\left\langle|B|^{2 \alpha} x, x\right\rangle^{r}+\left\langle\left|B^{*}\right|^{2(1-\alpha)} y, y\right\rangle^{r}}{2}\right)^{1 / r}
$$

(by Lemma 1)

$$
\leq\left(\frac{\left\langle|A|^{2 \alpha r} x, x\right\rangle+\left\langle\left|A^{*}\right|^{2(1-\alpha) r} y, y\right\rangle}{2}\right)^{1 / r}+\left(\frac{\left\langle|B|^{2 \alpha r} x, x\right\rangle+\left\langle\left|B^{*}\right|^{2(1-\alpha) r} y, y\right\rangle}{2}\right)^{1 / r}
$$

(by Lemma 3(a))

$\leq 2^{1-1 / r}\left(\frac{\left\langle|A|^{2 \alpha r} x, x\right\rangle+\left\langle\left|A^{*}\right|^{2(1-\alpha) r} y, y\right\rangle+\left\langle|B|^{2 \alpha r} x, x\right\rangle+\left\langle\left|B^{*}\right|^{2(1-\alpha) r} y, y\right\rangle}{2}\right)^{1 / r}$

(by the concavity of the function $f(t)=t^{1 / r}$ on $[0, \infty)$ ).

Thus,

$$
\begin{aligned}
& |\langle(A+B) x, y\rangle|^{r} \\
& \quad \leq 2^{r-2}\left(\left\langle\left(|A|^{2 \alpha r}+|B|^{2 \alpha r}\right) x, x\right\rangle+\left\langle\left(\left|A^{*}\right|^{2(1-\alpha) r}+\left|B^{*}\right|^{2(1-\alpha) r}\right) y, y\right\rangle\right) .
\end{aligned}
$$

Now, the desired inequality (9) follows from the fact that for every $T \in$ $B(H),\|T\|=\sup \{|\langle T x, y\rangle|: x, y \in H,\|x\|=\|y\|=1\}$.

Letting $x=y$ in the proof of Theorem 6 , it can be easily shown that

$$
w^{r}(A+B) \leq 2^{r-2}\left\||A|^{2 \alpha r}+|B|^{2 \alpha r}+\left|A^{*}\right|^{2(1-\alpha) r}+\left|B^{*}\right|^{2(1-\alpha) r}\right\| .
$$

In particular, if $A=B$, then the inequality (10) reduces to the inequality (6).

An important special case of Theorem 6 asserts that if $A$ and $B$ are normal, $\alpha=1 / 2$, and $r \geq 1$, then

$$
\|A+B\|^{r} \leq 2^{r-1}\left\||A|^{r}+|B|^{r}\right\| .
$$


Acknowledgements. The authors are grateful to the referee for his suggestions.

\section{References}

[1] R. Bhatia and F. Kittaneh, Norm inequalities for positive operators, Lett. Math. Phys. 43 (1998), 225-231.

[2] S. S. Dragomir, Some inequalities for the Euclidean operator radius of two operators in Hilbert spaces, Linear Algebra Appl. 419 (2006), 256-264.

[3] K. E. Gustafson and D. K. M. Rao, Numerical Range, Springer, New York, 1997.

[4] P. R. Halmos, A Hilbert Space Problem Book, 2nd ed., Springer, New York, 1982.

[5] G. H. Hardy, J. E. Littlewood, and G. Pólya, Inequalities, 2nd ed., Cambridge Univ. Press, Cambridge, 1988.

[6] F. Kittaneh, Notes on some inequalities for Hilbert space operators, Publ. Res. Inst. Math. Sci. 24 (1988), 283-293.

[7] -, Bounds for the zeros of polynomials from matrix inequalities, Arch. Math. (Basel) 81 (2003), 601-608.

[8] -, A numerical radius inequality and an estimate for the numerical radius of the Frobenius companion matrix, Studia Math. 158 (2003), 11-17.

[9] —, Numerical radius inequalities for Hilbert space operators, ibid. 168 (2005), 73-80.

[10] T. Yamazaki, On upper and lower bounds of the numerical radius and an equality condition, ibid. 178 (2007), 83-89.

Department of Mathematics

Science College for Girls

Dammam, Saudi Arabia

E-mail: m_el_haddad@yahoo.com
Department of Mathematics University of Jordan Amman, Jordan E-mail: fkitt@ju.edu.jo

Received June 11, 2006

Revised version June 18, 2007 